Rodríguez López, C. M., A. C. Wetten and M. J. WilkinSON (2004): Detection and quantification of in vitro-culture induced chimerism using simple sequence repeat (SSR) analysis in Theobroma cacao (L.). Theor Appl Genet 110: 157-166.

ROTTENBERG, A. (2000): Fertility of exceptional bisexual individuals in four dioecious plant species. Sex Plant Reprod 12: 219-221.

Rowland, D. L., R. GARNER and M. JEspersen (2002): A rare occurrence of seed formation on male branches of the dioecious tree, Populus deltoides. Am Mild Nat 147: 185-187.

SANTAMOUR, F. S. (1956): Hermaphroditism in Populus, pp. 28-30 in Proc $3^{\text {rd }}$ Northeastern For Tree Imp Conf, Ithaca, NY.

Slavov, G. T., S. Leonardi, J. BurczyK, W. T. Adams, S. H. Strauss and S. P. DiFAZIo (2009): Extensive pollen flow in two ecologically contrasting populations of Populus trichocarpa. Mol Ecol 18: 357-373.

Smulders, M. J. M., J. VAN Der Schoot, P. Arens and B. Vosman (2001): Trinucleotide repeat microsatellite markers for black poplar (Populus nigra L.). Mol Ecol Notes 1: 188-190.

STATSofT, INC. (2009): STATISTICA (data analysis software system), version 9.0. www.statsoft.com.

StebBins, G. L. (1958): The inviability, weakness, and sterility of interspecific hybrids. Adv Genet 9: 147-215.

SteTtLeR, R. F. (1971): Variation in Sex Expression of Black Cottonwood and Related Hybrids. Silvae Genet 20: $42-46$.

Stettler, R. F., H. D. Bradshaw JR., P. E. Heilman and T. M. HinckLey (1996): Biology of Populus and its implications for management and conservation. NRC Research Press, Ottawa.

VAN DER Schoot, J., M. PospíšKovÁ, B. Vosman and M. J. M. SMUlDERS (2000): Development and characterization of microsatellite markers in black poplar (Populus nigra L.). Theor Appl Genet 101: 317-322.

Von Melchior, G. H. (1967): Two cases of hermaphroditism on poplars of the section Aigeiros. Silvae Genet 16: $77-80$

\title{
Short Note: Development and characterization of 16 new polymorphic microsatellite loci for Schima superba (Theaceae)
}

\author{
By Hong-Yu Niu ${ }^{1,2)}$, WAn-Hui YE ${ }^{2)}$, Zheng-Feng WAnG ${ }^{2, *}$, , Ying Chen $^{2)}$, \\ Hong-Lin $\mathrm{CAO}^{2)}$, Lin-FAng WU ${ }^{2)}$ and ZHANG-Ming WANG ${ }^{2)}$
}

(Received $10^{\text {th }}$ October 2012)

\begin{abstract}
Schima superba is a common dominant tree species in evergreen broad-leaved forest in subtropical China. Despite its multiple usages in wood industry, reforestation and traditional Chinese medicine, its genetic diversity is poorly studied. To help studying its genetic diversity and structure in the future, after microsatellite enrichment and screening, we identified 16 microsatellites in S. superba. These markers showed polymorphism in three populations. The number of alleles per locus ranged from 3 to 32 with a mean of 14 . Within populations, the observed and unbiased expected heterozygosities ranged from 0.048 to 0.926 and from 0.048 to 0.949 , respectively. The newly developed 16 microsatellites will be useful for investigating the genetic diversity and structure from large scale patterns to fine-scale structures in this species.

1) College of Life Science, Central China Normal University, Wuhan 430079, P. R. China.

2) Key Laboratory of Vegetation Restoration and Management of Degraded Ecosystems, South China Botanical Garden, Chinese Academy of Sciences, Guangzhou 510650, P. R. China.

*) Corresponding author: Zheng-Feng Wang. South China Botanical Garden, Chinese Academy of Sciences, XingKe Road 723, TianHe Guangzhou 510650, P. R. China. Tel: +86-20-37252996, Fax: +86-20-37252615. E-Mail: wzf@scib.ac.cn
\end{abstract}

Key words: DHS plot, DNA enriched libraries, genetic marker, genetic variation, Hardy-Weinberg equilibrium, linkage disequilibrium, marker development, microsatellite, population genetics, reforestation, spatial genetic structure, subtropical China.

Schima superba Gardener \& Champ. (Theaceae) is a common evergreen fast growing pioneer tree species in south and east China. Its wood is hard and bug resistant and can be used in furniture and construction. Its root bark has been used as a traditional Chinese medicine, and stem extracts inhibit some cancers (XU et al., 2010). It has been widely used in reforestation and fire-preventing in plantations because its leaves are thick and contain high water content (YANG et al., 2008; LI et al., 2011). In large scales, it contains high variations in seed mass, leaf morphology and wood color among different sites and populations (ZHANG et al., 2004; WANG et al., 2011). In local scales, there were 2290 individuals with $\mathrm{DBH} \geq 1 \mathrm{~cm}$ recorded in 2005 census in the 20 ha Dinghushan forest dynamics plot (DHS plot, $\left.23^{\circ} 10^{\prime} 09^{\prime \prime}-23^{\circ} 10^{\prime} 25^{\prime \prime} \mathrm{N}, 112^{\circ} 32^{\prime} 05^{\prime \prime}-112^{\circ} 32^{\prime} 21^{\prime \prime E}\right)$ in Guangdong province, China, and they occupy different topographies with different age structure. Therefore, it would be interesting to know the contribution of genetic 
Table 1. - Characterization of 16 microsatellites for Schima superba. $T_{\mathrm{a}}$ : annealing temperature; $N_{\mathrm{a}}$ : number of alleles.

\begin{tabular}{|c|c|c|c|c|c|c|c|}
\hline Iocus & Repcat motit's & Primer sequetices $\left(5^{\prime}-3^{\prime}\right)$ & $\begin{array}{l}\text { Size range } \\
\text { (bp) }\end{array}$ & $T_{\mathrm{i}}\left(C^{\prime}\right)$ & $N_{0}$ & $\begin{array}{c}\text { 5'-fluorescence } \\
\text { label }\end{array}$ & $\begin{array}{c}\text { GenBank } \\
\text { Accession No. }\end{array}$ \\
\hline \multirow[t]{2}{*}{$\$ \$ 37$} & $(\mathrm{CN})_{1 !}$ & F: $\triangle$ GAGGCTACCOAGCANTG & $199-209$ & 52 & 5 & IIE:X & $J \times 448324$ \\
\hline & & R: GCACAGGICICAGAAAGCA & & & & & \\
\hline \multirow[t]{2}{*}{ SS38 } & $(\mathrm{AC})_{2}(\mathrm{IC})_{2}$ & F: IGGA IGCTCIGCTICTTC & $278-331$ & 57 & 23 & l:AM & $J \times 448325$ \\
\hline & & R: $\triangle$ GTGGTTGCCATTGACGG & & & & & \\
\hline \multirow[t]{2}{*}{$\$ \$ 39$} & $(\mathrm{~A})_{12}(\mathrm{CAN})_{\mathrm{K}}$ & F: TCCGAАGCA & $231-238$ & 5,5 & 8 & FАM & IX448326 \\
\hline & & R: A AGGAGGGGATGGITGI' & & & & & \\
\hline \multirow[t]{2}{*}{$\$ \$ 40$} & CACATT(CACACT) ${ }_{4} C A C A T T$ & F: TCCACCCATCAACCAATAG & $252-264$ & 52 & 3 & FAM & $\mathrm{JX} 448327$ \\
\hline & & R: AGTCACGATCCAMAGCAG & & & & & \\
\hline \multirow[t]{2}{*}{ SS41 } & Complex" & F: GCACСАТСТTCСТСТTT & $222-278$ & 55 & 32 & FAM &. $\mathrm{IX} 448.328$ \\
\hline & & R: AICAGCCAAGCAICCGTC & & & & & \\
\hline \multirow[t]{2}{*}{$S \$ 42$} & CAAGG(CAA) $\mathrm{CAG}(\mathrm{CAN})_{3} \mathrm{TGGCA}$ & F: IIAGCOICIGACACOACA & $261-282$ & 55 & 5 & FAM & $J \times 448329$ \\
\hline & & R: GGAACATGAATCCAAACC & & & & & \\
\hline \multirow[t]{2}{*}{$\$ S 43$} & $(C \Lambda)_{5}(\Lambda)_{5}$ & F: GTTCCGCTTATCGTCGTG & $208-233$ & 52 & 19 & FAM & $I \times 448330$ \\
\hline & & R: TTAGGGAACAATGCCATC: & & & & & \\
\hline \multirow[t]{2}{*}{$S S 44$} & $(\mathrm{CA})_{2 i}$ & F: ICCAACCGATAGAIGAGC & $251-348$ & 55 & 22 & l:AM & $J \times 448331$ \\
\hline & & R: CAAACCTACCOACGAAGC & & & & & \\
\hline \multirow[t]{2}{*}{$S S 45$} & CAATAA(CAA) & F: CAATAGCACCACCAGCAG & $193-203$ & 52 & 9 & IIEX & $\mathrm{JX} 448332$ \\
\hline & & R:GATIGCCAAGJGGIGICI & & & & & \\
\hline \multirow[t]{2}{*}{ SS46 } & $(\mathrm{CAA})_{4}(\mathrm{AGG})_{2}$ & F: GCGICICOAGIGACCAJ & $265-280$ & 55 & 6 & FAM & $.1 \times 448.333$ \\
\hline & & R: TAACTIGCCTCCCTCCTG & & & & & \\
\hline \multirow[t]{2}{*}{$\$ S 47$} & $(\mathrm{CA})_{1-1}$ & F: TAGGTAGGTGCTGGAGTAG & $212-224$ & 57 & 8 & FAM & $\mathrm{JX} 448334$ \\
\hline & & R: CAGGITIACACA]"MGGC & & & & & \\
\hline \multirow[t]{2}{*}{ SS48 } & МATAGANTG $(\Lambda \Lambda G)_{\varsigma}$ & F: GATAG $\triangle A G T A \Lambda A C A T C A G G C$ & $215-221$ & 52 & 3 & HEX & $\mathrm{JX} 448335$ \\
\hline & & R:GTTGCACAАGTTGTCC $\wedge \Lambda T$ & & & & & \\
\hline \multirow[t]{2}{*}{ SS49 } & $(\mathrm{ATG})_{7}(\mathrm{AG})_{i}$ & F: GATGATTCTCGGTTTCTTG & $207-219$ & 52 & 4 & $\mathrm{~F} A \mathrm{M}$ &. $\mathrm{TX} 448336$ \\
\hline & & R: GITATGITGI"IGGCGLTG & & & & & \\
\hline \multirow[t]{2}{*}{$\$ \$ 50$} & $(A G)_{21}$ & F: CACTGGIGTCACTGCITCLTG & $241-299$ & 57 & 29 & $1: A M$ & JX448337 \\
\hline & & R: CCTTTAGTGGANCGGCATC & & & & & \\
\hline \multirow[t]{2}{*}{$\$ \$ 51$} & $(\mathrm{AG})_{23}$ & FCAACGAGGAGIANAIGATG & $263-294$ & 55 & 19 & $\mathrm{l} A \mathrm{M}$ & $J \times 448338$ \\
\hline & & R: MAAGAGIGGAIACIGGIGI & & & & & \\
\hline \multirow[t]{2}{*}{ SS 52} & $(\mathrm{AG})_{1 \geq}$ & F: ATGGAAGAGTAAGGGGAATC & $190-296$ & 55 & 24 & lAM & $J \times 448339$ \\
\hline & & R: CCT"TAT"TAGl"IGGGAGC & & & & & \\
\hline
\end{tabular}

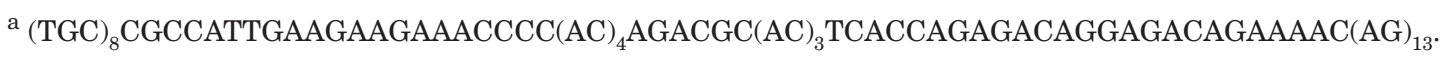

diversity to its phenotypic diversity in large scale and to its population persistence in small scale. However, up to now, its genetic variations are poorly known. In this work, we reported newly developed polymorphic microsatellites in order to better study Schima superba genetic variation in future.

Total genomic DNA was extracted, using a modified CTAB method, from one dry leaf tissue of $S$. superba in the DHS plot. The 300-1000 bp fragments digested from genomic DNA using restriction enzyme MseI [New England Biolabs (NEB), Massachusetts, USA] were ligated to $M s e I$ adaptors (MseI F: 5'-TACTCAGGACTCAT3' and MseI R: 5'-GACGATGAGTCCTGAG-3') using $\mathrm{T}_{4}$ DNA ligase (NEB). The digestion-ligation mixture was subsequently diluted 10 times, and $2 \mu \mathrm{L}$ of the diluted was used for PCR amplification using MseI-adaptor specific primers (5'-GATGAGTCCTGAGTAAN-3', i.e., MseI-N). Amplified products were then hybridized with 5'-biotinylated $(\mathrm{AG})_{15},(\mathrm{AAG})_{8},(\mathrm{AC})_{15}$ and $(\mathrm{AAC})_{8}$ probes.
Subsequent probe-bound DNA fragments were enriched by streptavidin-coated magnetic beads (NEB). The enriched fragments were recovered with PCR reaction using MseI-N as primer. The selected fragments were then ligated into the pGEM-T plasmid vector (Promega Corp, Wisconsin, USA) and transformed into the Escherichia coli DH5 $\alpha$ competent cells (Takara Bio Inc., Shiga, Japan). A PCR-based method (LUNT et al., 1999) was used to screen the recombinant clones. Ninety-three positive clones were identified and sequenced by Majorbio Biotech Co., LTD (Shanghai, China) with M13R or M13F as primers. Primers were designed using PREMIER 5.0 (PREMIER Biosoft International, Palo Alto, CA, USA) for the sequences that contained repeats motifs.

To assess the polymorphisms of the microsatellites, leaves were collected from 32, 32 and 28 randomly selected $S$. superba trees in Zhaoqing $\left(23^{\circ} 10^{\prime} 1^{\prime \prime} \mathrm{N}\right.$, $\left.112^{\circ} 32^{\prime} 21^{\prime \prime E}\right)$, Dongguan ( $\left.22^{\circ} 54^{\prime} 39^{\prime \prime N}, 114^{\circ} 13^{\prime} 21^{\prime \prime E}\right)$ and 
Table 2. - Genetic diversity of 16 loci in three populations of Schima superba. $N$ : sample size; $N_{\mathrm{a}}$ : number of alleles; $H_{\mathrm{o}}$ : observed heterozygosity; $H_{\mathrm{e}}$ : unbiased expected heterozygosity; $F$ : fixation index.

\begin{tabular}{|c|c|c|c|c|c|c|c|c|c|c|c|c|}
\hline \multirow{2}{*}{ Iocus } & \multicolumn{4}{|c|}{ POP-Zhaoging $(N=32)$} & \multicolumn{4}{|c|}{ POP-Dongguan $(N=32)$} & \multicolumn{4}{|c|}{ POP-Shavguan $(N=28)$} \\
\hline & $N_{u}$ & $H_{i}$ & $H_{*}$ & $F$ & $N_{c s}$ & $H_{a}$ & $H_{c^{\prime}}$ & $F^{\prime}$ & $\mathrm{N}_{u}^{\prime}$ & $H_{0}$ & $H_{\varepsilon}$ & $\mu$ \\
\hline $\operatorname{ss37}$ & 2 & 0.048 & 0.048 & -0.024 & 5 & 0.250 & 0.285 & $0.110^{*}$ & 5 & 0.560 & 0.598 & 0.044 \\
\hline $\operatorname{Ss} 38$ & 12 & 0.880 & 0.837 & -0.073 & 14 & 0.759 & 0.878 & 0.121 & 19 & 0.889 & 0.927 & 0.023 \\
\hline$\$ \$ 39$ & 6 & 0.148 & 0.418 & $0.639 *$ & 5 & 0.172 & 0.575 & $0.695 *$ & 5 & 0.370 & 0.766 & $0.507 *$ \\
\hline $\mathrm{SS} 40$ & 2 & 0.393 & 0.431 & 0.071 & 3 & 0.281 & 0.426 & 0.329 & 3 & 0.357 & 0.309 & -0.176 \\
\hline $\mathrm{SS41}$ & 12 & 0.320 & 0.863 & $0.622^{*}$ & 20 & 0.679 & 0.949 & 0.272 & 20 & 0.333 & 0.937 & $0.638^{*}$ \\
\hline $\mathrm{SS} 42$ & 4 & 0.179 & 0.260 & $0.302^{*}$ & 2 & 0.563 & 0.411 & -0.391 & 3 & 0.643 & 0.473 & -0.383 \\
\hline $\mathrm{SS43}$ & 15 & 0.467 & 0.853 & $0.444^{*}$ & 10 & 0.217 & 0.860 & $0.742^{*}$ & 10 & 0.304 & 0.887 & $0.649 *$ \\
\hline $\mathrm{SS} 44$ & 12 & 0.500 & 0.802 & $0.365^{*}$ & 15 & 0.357 & 0.916 & $0.603^{\text {* }}$ & 9 & 0.292 & 0.839 & $0.645^{\circ}$ \\
\hline $\mathrm{SS45}$ & 7 & 0.519 & 0.628 & 0.159 & 8 & 0.813 & 0.804 & -0.027 & 7 & 0.769 & 0.766 & -0.024 \\
\hline $\mathrm{SS46}$ & 5 & 0.667 & 0.730 & 0.070 & 6 & 0.613 & 0.797 & 0.218 & 6 & 0.750 & 0.807 & 0.054 \\
\hline $5 S 47$ & 7 & 0.640 & 0.763 & $0.144^{*}$ & 6 & 0.806 & 0.682 & -0.202 & 5 & 0.520 & 0.699 & 0.241 \\
\hline SS48 & 3 & 0.577 & 0.551 & -0.067 & 3 & 0.813 & 0.637 & -0.296 & 3 & 0.692 & 0.541 & -0.305 \\
\hline $\mathrm{SS} 49$ & 4 & 0.679 & 0.588 & -0.176 & 4 & 0.769 & 0.645 & -0.216 & 4 & 0.880 & 0.633 & -0.419 \\
\hline$S S 50$ & 17 & 0.926 & 0.931 & -0.014 & 20 & 0.813 & 0.920 & 0.103 & 19 & 0.875 & 0.936 & 0.045 \\
\hline SS51 & 13 & 0.571 & 0.894 & $0.349^{*}$ & 12 & 0.281 & 0.891 & $0.679^{*}$ & 12 & 0.320 & 0.869 & $0.624 *$ \\
\hline $\operatorname{sS} 52$ & 12 & 0.704 & 0.898 & 0.202 & 19 & 0.581 & 0.911 & $0.352 *$ & 17 & 0.808 & 0.925 & 0.109 \\
\hline
\end{tabular}

* $(P<0.05)$ indicates a significant deviation from Hardy-Weinberg equilibrium after Holm's sequential Bonferroni correction.

Shaoguan $\left(24^{\circ} 46^{\prime} 53^{\prime \prime} \mathrm{N}, 113^{\circ} 36^{\prime} 16^{\prime \prime} \mathrm{E}\right)$ populations in Guangdong province, respectively. PCR amplifications were performed in a $20 \mu \mathrm{L}$ reaction mixture containing $20 \mathrm{mM}$ Tris- $\mathrm{HCl}\left(\mathrm{pH}\right.$ 8.4), $100 \mathrm{mM}\left(\mathrm{NH}_{4}\right)_{2} \mathrm{SO}_{4}, 3 \mathrm{mM}$ $\mathrm{MgCl}_{2}, 0.4 \mathrm{mM}$ dNTPs, $0.4 \mu \mathrm{M}$ each primer ( 5 ' end fluorescent dye labeled with FAM or HEX), $50 \mathrm{ng}$ of genomic DNA, and 1 U Taq polymerase (Takara). The amplification program was $95^{\circ} \mathrm{C}$ for 5 min, 35 cycles of $94^{\circ} \mathrm{C}$ for $30 \mathrm{~s}$, optimized annealing temperature for $30 \mathrm{~s}$ and $72{ }^{\circ} \mathrm{C}$ for $45 \mathrm{~s}$, and a final extension at $72{ }^{\circ} \mathrm{C}$ for $10 \mathrm{~min}$. PCR products were electrophoresis-analyzed on ABI 3730 sequencer (Applied Biosystems Inc., California, USA) and the lengths of them were analyzed by ABI GeneMapper Software Version 3.7. Number of alleles $\left(N_{\alpha}\right)$, observed and unbiased expected heterozygosities $\left(H_{o}, H_{e}\right)$ and fixation index $(F)$ were obtained using GenAlEx 6.2 (PEAKall and SMouse, 2006). Deviation from Hardy-Weinberg equilibrium (HWE) and genotypic linkage disequilibrium among all pairs of loci in each population were analyzed with GENEPOP 3.4 (RAYMOND and Rousset, 1995). Significance levels were adjusted using Holm's sequential Bonferroni correction (HoLM, 1979) implemented in $R$ "stats" package ( $R$ DEVELOPMENT CoRe TEAM, 2011).

A total of 16 polymorphic microsatellite loci were amplified with clear and stable polymorphism (Table 1). Overall, alleles per locus varied from 3 to 32 with a mean of 14 (Table 1). Within populations, $H_{0}$ ranged from 0.048 to 0.926 , and $H_{e}$ from 0.048 to 0.949 (Table 2). Four loci showed significant deviation from HWE in one population each, one locus in two and four loci in all three populations (Table 2). No consistently significant linkage disequilibrium was detected among all pairs of loci within each population, indicating the independence of these 16 microsatellite markers.
The polymorphic microsatellites developed here will be powerful genetic tools for studying the genetic diversity and structure of $S$. superba. Such genetic information will help to classify the germplasm resource, understand adaptation, and then establish conservation policy for $S$. superba. They will also be useful in studying finescale spatial genetic structure in DHS plot, which is important in analyzing population dynamics and persistence in the local population (EPPERSON, 1992).

\section{Acknowledgments}

This work was supported by Foreign Exchange Program National Founder (31061160188), Knowledge Innovation Program of the Chinese Academy of Sciences (KSCX2-EW-Z, KSCX2-EW-J-28), Chinese Forest Biodiversity Monitoring Network.

\section{References}

EPPERSON, B. K. (1992): Spatial structure of genetic-variation within populations of forest trees. New Forests 42: 257-278.

Holm, S. (1979): A simple sequentially rejective multiple test procedure. Scandinavian Journal of Statistics 6 65-70.

Li, P., Z. L. Huang, Y. C. Xiang and H. Ren (2011): Survival, growth and biomass of Acacia auriculiformis and Schima superba seedlings in different forest restoration phases in Nan'ao island, south China. Journal of Tropical Forest Science 23: 177-186.

Lunt, D. H., W. F. Hutchinson and G. R. Carvalho (1999): An efficient method for PCR-based isolation of microsatellite arrays (PIMA). Molecular Ecology 8: 891-894. 